CLAUDIA GARDUÑO GARCÍA

FACULTAD DE ARQUITECTURA

UNIVERSIDAD NACIONAL AUTÓNOMA DE MÉXICO,

CIUDAD DE MÉXICO, MÉXICO.

CLAUDIAGARDUNO@POSGRADO.UNAM.MX

GABRIELA CONTRERAS MARTÍNEZ

CIUDAD DE MÉXICO, MÉXICO.

COMAGABRIELA@GMAIL.COM

ANGÉLICA RUBí RUIZ TULE

CIUDAD DE MÉXICO, MÉXICO.

ANGIIETULEE@GMAIL.COM

LUCY ATRI PENHOS

CIUDAD DE MÉXICO, MÉXICO.

LUCYATRIP94@GMAIL.COM

IVÁN RODRIGO LÓPEZ QUINTANA

CIUDAD DE MÉXICO, MÉXICO.

IVANRODRIGOLOPQUI@GMAIL.COM
Fecha de recepción: 26/01/2021

Fecha de aceptación: 5/06/2021

Cómo citar: Garduño, C., Contreras, G.,

Ruiz, A., Atri, L. \& López, I. (2021).

TiUD 2020: la emergencia de relaciones

significativas durante el distanciamiento social

RChD: creación y pensamiento, 6(10), 1-19.

https//doi.org/10.5354/0719-837X.2021.60878

Revista Chilena de Diseño,

RChD: creación y pensamiento

Universidad de Chile

2021, 6(10).

http://rchd.uchile.cl

\section{TiUD 2020: la emergencia de relaciones significativas durante el distanciamiento social}

\author{
TiUD 2020: The Emergence of Meaningful Relationships During \\ Social Distancing
}

Resumen. En el 2020, la batalla contra el virus Covid-19 fue la emergencia común que perturbó todos los ámbitos de la vida humana; y que precipitó la transición de la educación hacia la digitalización. El Taller Interuniversitario de Diseño (TiUD) enfrentó el desafío de sostener sus atributos y objetivos, planteados sólidamente desde su fundación en 2018: el Diseño como una herramienta transformadora hacia futuros justos y sostenibles, aprendido a través de experiencias profundas en contextos reales, que permiten a las estudiantes asumirse individual y colaborativamente como agentes de cambio en sus comunidades. Para evaluar el cumplimiento de estos objetivos e identificar posibles ventajas y desventajas del TiuD en línea, el equipo investigador indagó en las percepciones de docentes y estudiantes mediante una metodología mixta (revisión de documentos del TiUD, encuestas, entrevistas grupales y observación participante). Se concluye que, aunque éste no es percibido como el mejor esquema, el TiUD virtual permitió el desarrollo de relaciones significativas y de experiencias profundas de aprendizaje-enseñanza que responden al contexto. Sugieren que la versión óptima combinaría sesiones virtuales y presenciales. Especialmente, se identifica que, dado que la facilitación de la socialización es el atributo más valorado por las estudiantes, sería beneficioso incluirla en cualquier experiencia educativa durante el distanciamiento social.

Palabras clave: activismo en Diseño, Covid-19, Diseño para las transiciones, educación en Diseño, relaciones significativas

\begin{abstract}
During 2020, the struggle against Covid-19 virus became the common emergency that disrupted all spheres of human life; it also pushed the transition of education to digitalization. The Inter-university Design Workshop (TiUD by its acronym in Spanish) faced the challenge of sustaining its solidly framed attributes and objectives since its foundation in 2018: Design as a transformational tool towards just and sustainable futures, learned through deep experiences in real contexts, which enable students to assume their roles as agents of change in their communities both individually and collectively. In order to evaluate the fulfilment of these goals and to identify possible advantages and disadvantages of TiuD's online version, the research team inquired into the perceptions of teachers and students through a mixed methodology (revision of TiUD's documents, polls, group interviews, and participant observation). It is concluded that, although this was not perceived as the best mode, the online TiUD enabled the development of meaningful relationships and deep learning-teaching experiences that respond to the context, suggesting that its optimal version would combine virtual and in-person sessions. It was especially identified that, given that the facilitation of socialization was perceived as TiUD's most valuable attribute, in times of social distancing, it might be beneficial to include these dynamics in every educational experience.
\end{abstract}

Keywords: Covid-19, Design activism, Design education, Design for transitions, meaningful relationships 
1. El equipo de investigadoras que produjo el presente artículo se une a la lucha contra la brecha de género en los estudios del Diseño. Por este motivo, la redacción del artículo prioriza a las participantes de la investigación.
Nota de los/las autores/as

Esta investigación no habría sido posible sin el apoyo del Consejo Nacional de Ciencia y Tecnología (CONACYT) a través de las becas nacionales para las estudiantes del Posgrado en Diseño Industrial de la Universidad Nacional Autónoma de México (PDI). Agradecemos el apoyo de nuestras colegas en el PDI, quienes se tomaron el tiempo de leer versiones anteriores. Toda nuestra gratitud a las diseñadorasdocentes del Taller Interuniversitario de Diseño (TiUD): Escuela de Diseño del Instituto Nacional de Bellas Artes (EDINBA) (Itzaé García Vera y Omar Israel Mendoza Rosas), Centro de Investigaciones en Diseño Industrial de la Universidad Nacional Autónoma de México (Diego Alatorre Guzmán), Universidad Autónoma Metropolitana, Unidad Azcapotzalco (Sandra Molina Mata, Ana Helena Hernández Palomino y Edrei Ibarra Martínez), Universidad Iberoamericana (Renee Harari Masri y Andrea Soler Guitián) y Tecnológico de Monterrey Campus Ciudad de México (Inés Álvarez-Icaza Longoria y Lucero Donají de la Huerta Santaella) y muy especialmente a todas las estudiantes que participaron en la versión pandémica del TiUD.

\section{Introducción}

Si bien la situación actual podría compararse con la ocasionada por la gripe española (Rossi et al., 2020), también es cierto que el mundo de hoy es muy distinto, entre otras cosas porque la interconexión global ha causado que, por primera vez en la historia, la humanidad entera enfrente el mismo reto al mismo tiempo (Dussel, 2020). En el último siglo, el Diseño ha transitado un largo camino, desde su concepción original como una herramienta para la expansión industrial (Buchanan, 1992; Bravo \& Bohemia, 2020). De ahí que Rossi et al. (2020), en su análisis de contribuciones (académicas) del Diseño a la emergencia global por Covid-19, señalen que ésta puede considerarse el mayor problema a enfrentar por una generación entera de diseñadoras ${ }^{1}$.

La pandemia provocó la aceleración de varios cambios tecnológicos y sociales que ya estaban en curso, pero que, de otra manera, hubieran implicado más años en concretarse (Rossi et al., 2020; Harari, 2020). En el ámbito educativo, en contextos donde existía la infraestructura tecnológica que lo permitiera, el uso de la tecnología dejó de ser opcional (Bernardo \& Duarte, 2020). Por años, aunque la transición hacia la educación a distancia a través de medios digitales era posible, había sido vista con recelo.

Aunque se habían identificado algunas ventajas, como la eliminación de barreras para la participación de las estudiantes, o el acceso a una gran cantidad de información (Arkoful \& Abaidoo, 2014, citado en Bernardo \& Duarte, 2020), también existen algunas desventajas importantes, como la falta de interacción social y el requerimiento de ciertas habilidades y actitudes (Hameed et al., 2008, citado en Bernardo \& Duarte, 2020). Algunas investigaciones sugieren que una modalidad mixta puede resultar más inclusiva (Wedgwood, 2012, citado en Bernardo \& Duarte, 2020), pero en el 2020 , la pandemia obligó a las instituciones educativas de algunos países a poner en práctica modalidades de educación a distancia exclusivamente.

En un estudio realizado en Pakistán por Adnan \& Anwar (2020), donde buscaban conocer la efectividad de la educación en línea, así como identificar sus retos y obstáculos desde la perspectiva de las estudiantes de educación superior, se concluye que la educación en línea no puede producir los resultados deseados en contextos donde la mayoría de las estudiantes carecen de acceso a internet, por razones técnicas o económicas. Adicionalmente, encontraron que las debilidades más frecuentemente mencionadas por las estudiantes se refieren a la falta de interacción cara a cara con las instructoras, el tiempo de respuesta y la falta de socialización tradicional.

Bernardo \& Duarte (2020) realizaron un estudio con el objeto de caracterizar las experiencias y percepciones de las docentes que impartieron cursos en línea durante la pandemia, en contextos donde esto era posible, en instituciones de educación superior y en áreas relacionadas con el Diseño. Las autoras identificaron algunas ventajas, como la posibilidad de combinar la enseñanza sincrónica con el aprendizaje asincrónico y la expansión del ambiente educativo. No obstante, identificaron dos graves retos o desventajas: la falta de control que la docente tiene sobre el ambiente (incluye las responsabilidades de las estudiantes en sus casas y la conectividad deficiente, entre otras), y la degradación de las relaciones humanas. En línea con lo observado por Adnan \& Anwar (2020), también 
concluyen que, aunque las docentes fueron capaces de impartir sus cursos durante la emergencia, la tecnología actual no provee algunos beneficios del salón de clases, como oportunidades de enseñanza y aprendizaje más variadas, y conexiones humanas más cercanas.

Dentro de las investigaciones citadas para este estudio, es especialmente importante rescatar que durante la pandemia, las instituciones de educación superior y las escuelas de Diseño, enfrentan dos grandes retos, igualmente importantes: transferir conocimientos de manera virtual; y cumplir con su rol socializador. Además de las percepciones de estudiantes (Adnar \& Anwar, 2020) y docentes (Bernardo \& Duarte, 2020), esta noción es sustentada por estudios como el desarrollado por Wilcox et al. (2006), donde encontraron que desarrollar amistades, a varios niveles de profundidad con compañeras de clase y con tutoras, es crucial para la retención de estudiantes, y concluyen que debería darse el mismo peso a la integración de las estudiantes en el mundo académico y en el mundo social de la universidad.

Otras autoras, como Martha Nussbaum (2006; 2010) y Boni et al. (2012), abogan por valorar a las instituciones de educación superior especialmente por su rol social y su capacidad transformadora; argumentan que la importancia de incluir estos roles, como objetivos específicos en las aulas de estudio, radica en la formación de ciudadanas con habilidades cosmopolitas como base para la formación de sociedades democráticas. Con base en el trabajo de Nussbaum, Walker et al. (2009) afirman que para la formación de profesionistas comprometidas con la justicia social es crucial el desarrollo de dos capacidades: la capacidad de ser un agente de cambio y la capacidad de afiliación. Más recientemente, en el marco de la conformación de la Agenda 2030 para el Desarrollo Sostenible, la Organización de las Naciones Unidas para la Educación, la Ciencia y la Cultura ([UNESCO], 2019) ha reconocido la creciente tendencia de jóvenes estudiantes a interesarse en tomar medidas para incidir en problemáticas complejas como la violencia armada y/o el cambio climático. Esto pone de relieve la importancia de comprender mejor el rol que puede desempeñar la educación en la detonación de la participación transformadora. Concluyen que, para que la alumna emprenda una acción o modifique su comportamiento, necesita reconocer que existe una brecha entre su realidad y la realidad de otras personas, así como llevar a cabo un proceso de internalización al conectarse con ellas a nivel cognitivo y emocional.

El Taller Interuniversitario de Diseño (TiUD) es un curso-taller experimental desarrollado en la Ciudad de México desde 2018. A través de diversas experiencias de aprendizaje, parecidas a las sugeridas por la UNESCO (2019), y desde los estudios en Diseño a nivel licenciatura, persigue objetivos y visiones similares a las propuestas por Nussbaum $(2006$; 2010) y Walker et al. (2009). Estas características diferencian al TiUD de otras asignaturas, y representan retos particulares aún en su versión presencial, que se vieron complejizados por el confinamiento obligatorio del año 2020.

\section{Objetivos y pregunta de investigación}

Esta investigación tuvo por objeto conocer los atributos del TiUD originados en cada una de sus diferentes versiones, con la finalidad de identificar aquellos que perduraron en la versión virtual, y otros posibles que pudieron 
2. El Instituto Tecnológico y de Estudios

Sueriores de Monterrey (ITESM) $\left(4^{\circ}\right)$, la

Universidad Nacional Autónoma de México

(UNAM) $\left(17^{\circ}\right)$ y la Universidad Autónoma

Metropolitana (UAM) $\left(21^{\circ}\right)$ aparecen listadas

dentro de las mejores universidades de América

Latina del Latin American University Rankings

2020 de Times Higher Education. De acuerdo con

el periódico El Universal (2019), entre las mejores

universidades de México se incluyen: UNAM $\left(1^{\circ}\right)$,

Universidad Iberoamericana (IBERO) $\left(5^{\circ}\right)$, UAM

$\left(6^{\circ}\right)$. El periódico Reforma (Rezc, 2020), por su parte, considera que las mejores universidades de la Ciudad de México incluyen al ITESM (3), la IBERO (4), la UNAM (6) y la UAM (7) y en su ranking por área de estudios muestran en arquitectura, UNAM (1), IBERo (2), ITESM (4), UAM (6) y en Diseño gráfico, Escuela de Diseño del Instituto Nacional de Bellas Artes (EDINBA) (1), UNAM (2), UAM (5), IBERO (6) y CENTRO (11). No existe el ranking para Diseño industrial (en Mextudia, 2020). surgir a pesar del confinamiento, lo que brindaría información para una versión ideal en semestres posteriores.

Consecuentemente, la pregunta de investigación es: ¿cuáles son los atributos del TiUD presencial y cómo se modificaron en la versión a distancia en línea, obligada por la pandemia por Covid-19?

Fue necesario revisar los documentos de ésta y las versiones pasadas del TiUD; así como indagar la percepción de las estudiantes y docentes mediante diferentes instrumentos de investigación. El estudio permitió identificar ventajas y desventajas de esta modalidad, gracias a las cuales se derivaron recomendaciones para el logro futuro de una versión óptima del taller. También se identificó que, durante la pandemia, la promoción de relaciones significativas posee un rol muy valorado para la educación.

Taller Interuniversitario de Diseño (TiUD)

El TiUD es una iniciativa del autodenominado grupo Diseño Interuniversitario, planteada a raíz del nombramiento de la Ciudad de México como "Capital Mundial del Diseño 2018" (nombre completo [WDC CDMX], 2018) y como Ciudad Creativa de la UNESCO. Como antecedente, cabe mencionar que, hacia finales de $\mathbf{2 0 1 6}$, el hoy desaparecido Laboratorio para la Ciudad (LabCDMX), que representaba el área creativa del gobierno de la CDMX, convocó a diseñadoras provenientes de distintas instituciones y sectores (público, privado, onGS y academia) a participar en mesas de trabajo orientadas a colaborar en la planificación del programa de WDC 2018. Posteriormente, en 2017 se conformaron grupos de trabajo por áreas de interés, y de ahí emergió un grupo de Diseño y Educación, el cual se autodenomina como Diseño Interuniversitario.

El grupo estuvo conformado principalmente por diseñadoras-docentes, nombradas así en este artículo debido a la experiencia que tienen tanto en la práctica del Diseño como en su enseñanza, dentro de algunas de las mejores universidades de la $\mathrm{CDMX} \mathrm{X}^{2}$ y que conciben al Diseño como una poderosa herramienta de cambio. Las diseñadoras-docentes hallaron en la colaboración interuniversitaria de Diseño un medio para potenciar el Diseño socialmente responsable y hacer del WDC 2018 un hito en la historia del Diseño en México. No obstante, ante la complicación burocrática que significaba desarrollar dicha colaboración de manera oficial (especialmente cuando la ciudad y las universidades se recuperaban de los daños del sismo del 19 de septiembre de 2017), las docentes optaron por programar sus materias para los miércoles e impartir el curso de manera colaborativa y extraoficial.

En un contexto donde la educación universitaria en Diseño industrial fue precedida y todavía se encuentra enormemente influenciada por la inserción del Diseño en la industria (Fernández, 2006), hay que señalar que el punto de partida del TiUD fue el Diseño para la activación social. Aquí se recuperó una definición dada por el grupo en la que se plantea "al Diseño como una herramienta con un potencial inexplorado para imaginar y sobre todo materializar colectivamente nuevos conceptos urbanos que propicien la visión de una ciudad enfocada en el bienestar común" (TiUD 2018). Toma 
como inspiración el trabajo de Alastair Fuad-Luke (2009)³ y está en diálogo constante con Diseño Activista ${ }^{4}$. En el marco de WDC 2018, y con una fuerte influencia del trabajo desarrollado por el LabCDMx en torno al derecho a la ciudad, el TiUD originalmente adoptó el concepto de lo común desde la perspectiva de David Harvey (2012, 161, citado en Estalella et al., 2013), según quien la colocación de "cuerpos humanos en este lugar [la calle], convierte el espacio público, en un procomún político" (p.39). No obstante, en su objetivo principal "que las estudiantes puedan asumirse como agentes de cambio en su comunidad a través del ejercicio del Diseño" (TiUD, 2018, p.9) y en su metodología, a través del desarrollo de proyectos en equipos interuniversitarios, que estuvieran enfocados en atender problemáticas reales de tipo social y localizadas en la Ciudad de México, también se alinea con la visión de Elinor Oström (2002)5, y con la desarrollada por el Laboratorio del procomún de Medialab-Prado (Madrid), de acuerdo con lo reportado por Estalella et al. (2013) .

Adopta un nombre distinto en cada universidad. La primera edición del TiUD se llevó a cabo entre agosto y diciembre, y tomó como sede neutral la de WDC: Espacio CDMX. Colaborativamente, todas las diseñadoras-docentes impartieron el mismo curso para las cerca de 70 alumnas, provenientes de tres universidades públicas: Escuela de Diseño del Instituto Nacional de Bellas Artes, el Centro de Investigaciones en Diseño Industrial de la Universidad Nacional Autónoma de México, la División de Ciencias y Artes para el Diseño de la Universidad Autónoma Metropolitana (Unidad Azcapotzalco); y tres universidades privadas: la Escuela de Arquitectura, Arte y Diseño del Tecnológico de Monterrey (Campus Ciudad de México), el Departamento de Diseño de la Universidad Iberoamericana y la Licenciatura en Diseño Industrial de CENTRO?.

El TiUD 2018 logró satisfacer tanto las expectativas de las estudiantes como de las docentes, y ganó la aprobación de las autoridades universitarias, tras lo cual se determinó su continuidad como una colaboración extraoficial. En su iteración de $2019^{8}$, el TiUD realizó algunas modificaciones, como la implementación de un fin de semana intensivo al inicio del curso, cinco temas como posibles puntos de partida y la regla de que al menos un integrante de cada equipo interuniversitario tenía que estar vinculado con la comunidad o contexto a explorar. Tras sus dos primeras ediciones, el TiUD se consolidó como una estrategia que contribuye a transformar la práctica del Diseño en México, al provocar experiencias profundas de aprendizaje que permiten a las estudiantes interuniversitarias identificar, activar y construir lo común a partir de tres objetivos particulares?.

1. Que las alumnas comprendan el potencial transformador del Diseño.

2. Que las alumnas se asuman como agentes de cambio en sus comunidades.

3. Que las alumnas se asuman colaborativamente como un gremio de Diseño para la activación social y la transición hacia la sostenibilidad.
3. Alastair Fuad-Luke (2009) define activismo en Diseño como: el pensamiento, la imaginación y la práctica del Diseño aplicada de forma consciente o inconsciente a la creación de una contra narrativa orientada a generar y balancear el cambio positivo; ya sea social, institucional, ambiental y/o económico (p. 27, traducción propia).

4. Diseño Activista es otro de los grupos de trabajo que surgió a partir de la planificación de WDC 2018. Para saber más sobre su trabajo, visitar: https://disenoactivista.mx/

5. Quien identificó que, para que lo común sea sostenible, debe adaptarse al contexto local.

6. Entender al procomún experimental como "un objeto epistémico a través del cual quienes se reúnen en torno a él problematizan todo tipo de dominios, prácticas y formas de organización social; ya sea la empresa, el cuerpo, la memoria o la ciudad..." (Lafuente \& Corsín Jiménez, 2010, en Estalella et al., 2013, p. 31), donde el procomún se convierte en el verbo procomunizar, "que es tomar en bien común objetos diversos mediante la experimentación ciudadana" (Estalella et al., 2013, p. 32).

7. Después de la primera edición, CENTRO fue la única universidad que cesó su participación debido a que la diseñadora-docente que representaba a esta escuela dejó de trabajar ahí. Esto muestra una clara desventaja de que la colaboración sea extraoficial, pero queda fuera del alcance de esta investigación.

8. El TiUD 2019 fue postulado al premio Diseña México, en la categoría Diseño de experiencias, y fue acreedor de una mención honorífica.

9. Estos objetivos no han sido enunciados puntualmente por el TiUD; el equipo de investigación en este estudio sintetiza así lo descrito en los materiales oficiales del TiUD.

TiUD 2020: La emergencia (de lo) común

En esta ronda iterativa, el equipo de diseñadoras-docentes buscaba profundizar las bases teóricas del TiUD; pero a partir del 16 de marzo, fecha 
10. "México está dentro del $25 \%$ de los países con mayores niveles de desigualdad en el mundo.. Con base en las últimas cifras de Forbes... las 10 personas más ricas de México acumulan la misma riqueza que el 50\% más pobre del país" (Vázquez Pimentel et al., 2018, p. 2).

11. Para el año 2018, en la Ciudad de México el $28,8 \%$ de la población vivía en situación de pobreza moderada, el 1,7\% en pobreza extrema, el $28 \%$ en situación de vulnerabilidad por carencias sociales, el 7,8\% en situación de vulnerabilidad por ingresos, y solamente el 33,3\% vivía en situación de no pobreza ni vulnerabilidad (CONEVAL, 2020, p. 17)

12. De acuerdo con la "Guía para la Inversión 2019 CDMX Secretaría de Desarrollo Económico SEDECO" el $99.8 \%$ de los hogares de la Ciudad de México tiene internet de banda ancha (Secretaría de Desarrollo Económico, 2019, p. 31); en contraparte, solamente el $53 \%$ de la población nacional tiene acceso a internet, de acuerdo con la información dada a conocer en el coloquio "La Universidad y su Futuro", organizado por PNUD México y el IISUE de la UNAM. Además, en el contexto de la pandemia, el PNUD "estima que, en México, más de medio millón de alumnas de licenciatura y posgrado podrían abandonar sus estudios... cifras equivalentes al $15 \%$ de todas las alumnas del sistema universitario del país. Sólo en la UNAM, la Secretaría General estima que 72 mil jóvenes podrían dejar sus estudios" (López \& Romero, 2020). en la que el Gobierno de México ordenó el cierre de toda actividad no esencial, incluidos centros educativos, la planificación de la tercera edición se llevó a cabo en un ambiente de absoluta incertidumbre.

Tras la participación de algunas diseñadoras-docentes en un taller impartido en línea por Terry Irwin, el Tiud se inclinó hacia el Diseño para las transiciones (Irwin, 2015; Irwin et al., 2020), que fue incluido como uno de los tres ejes teórico-metodológicos de la edición de 2020, junto con la sustentabilidad y el activismo (TiUD, 2020). En línea con Bernardo \& Duarte (2020), podría pensarse que la profundización de las bases teóricas, en conjunto con la metodología y herramientas presentadas por Irwin, aseguraba o al menos incrementaba las posibilidades de que el TiUD en su versión virtual cumpliera con el objetivo de que las alumnas comprendieran el poder transformador del Diseño. No obstante, los objetivos relacionados con la expansión de las capacidades para la ciudadanía cosmopolita (Nussbaum, 2006; Walker et al., 2009) aparentaban ser retos más complejos. En este sentido, las diseñadoras-docentes se cuestionaban: ¿cómo lograr que las estudiantes se asuman como agentes de cambio en sus comunidades cuando no es posible interactuar con las comunidades? ¿Cómo lograr que las estudiantes se asuman colaborativamente como un gremio de Diseño, cuando no es posible que se relacionen de manera presencial?

Aquí habría que enfatizar que la activación de lo común es simultáneamente una de las propuestas más loables y retadoras del TiUD, en cualquier ocasión que el curso-taller se lleva a cabo en uno de los países más desiguales del mundo ${ }^{10}$. El TiUD puede entenderse en sí mismo como un proyecto de Diseño Activista, pues al incentivar la convivencia horizontal entre todas las personas, en una ciudad donde el $66 \%$ de la población vive entre la pobreza extrema y la vulnerabilidad por ingreso ${ }^{11}$ (ver Figura 1), abre un espacio para la generación de una contranarrativa. Entonces, si por un lado se especulaba que la pandemia, al afectar simultáneamente la vida de todas las personas del planeta, abría un espacio para la empatía, la conversación y la reflexión, por el otro lado, quedaba claro que en sociedades polarizadas como la mexicana, una pandemia se enfrenta desde condiciones socio-económicas muy distintas. En el TiUD virtual, las circunstancias de algunos estudiantes podrían ser especialmente adversas y la clase interuniversitaria podía ser el reflejo fiel de una sociedad severamente fragmentada ${ }^{12}$. En línea con lo identificado por Freire et al. (2020), las diseñadoras-docentes del TiuD se encontraron con la necesidad de Diseñar durante y para la emergencia, en un contexto especialmente crítico.

El proceso de Diseño del TiUD 2020 incluyó un conversatorio titulado La Emergencia (de lo) Común; hace alusión a la posibilidad de que la emergencia común permitiera que surgiera lo común, donde participaron Mariana Salgado (del Ministerio del Interior de Finlandia y conductora del podcast Diseño y Diáspora) y Ricardo Bello Bolio (Director General de Investigación e Innovación en el Estado de Yucatán, México). La conversación se detonó a partir de un texto-provocación que concluía de la siguiente manera: 


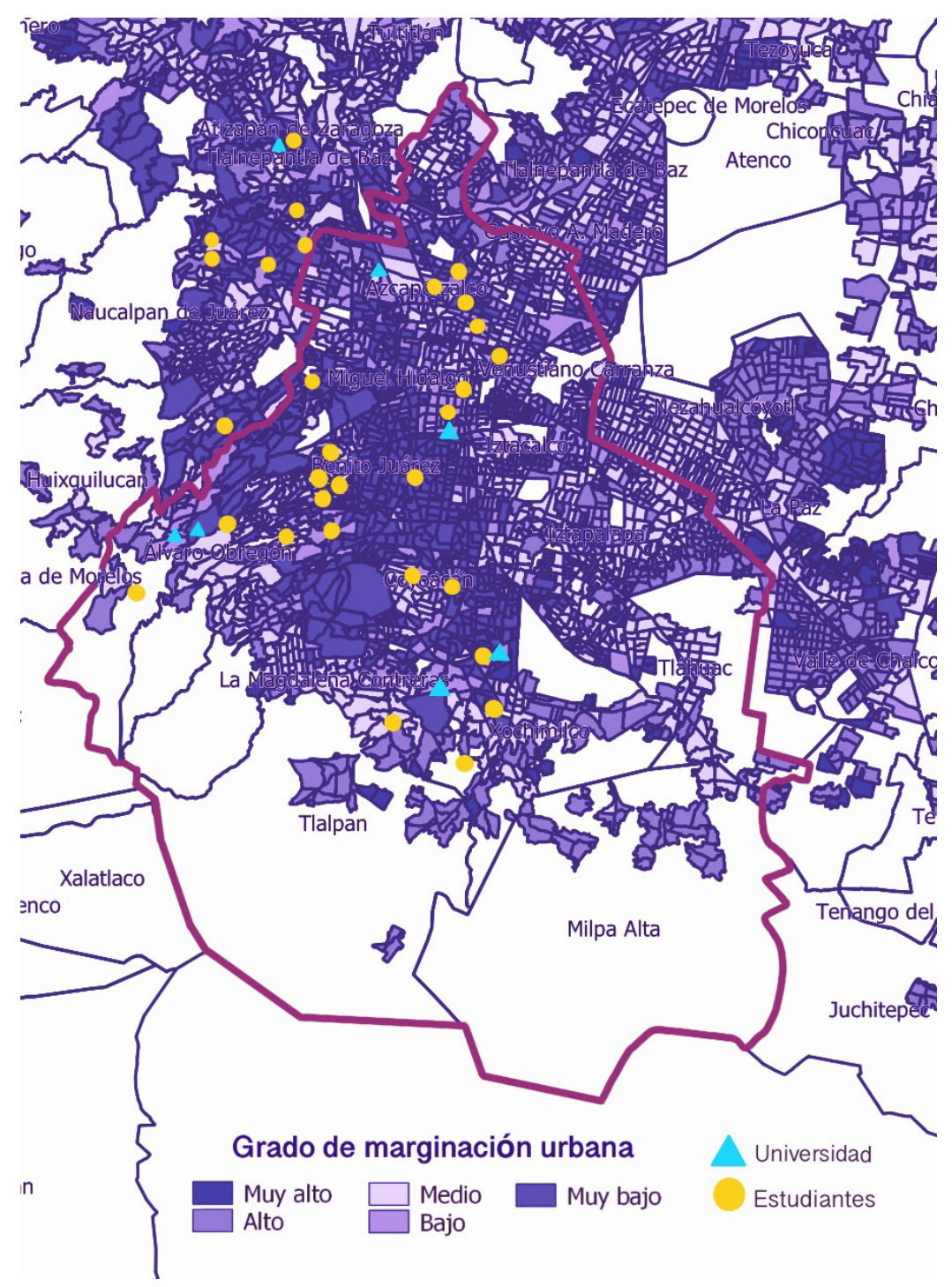

En el TiUD como en cualquier otro foro, la emergencia será necesariamente, la más evidente de las comunalidades. En este momento en el que el presente mismo es tan incierto, nos sentimos obligados a reflexionar y a dialogar sobre el futuro común que queremos construir:

¿Cómo podría ayudarnos "la emergencia común" a identificar aquello que vale la pena sostener?

¿Cómo podríamos sostener "lo común" durante un periodo de emergencia? ¿Qué podemos activar en un mundo en emergencia para una transformación sostenible? ¿Qué necesitamos para generar o construir el ambiente propicio para la transformación? ¿Qué debemos transformar en un mundo en emergencia? (TiUD, 2020a)

Entre otras reflexiones interesantes, Bello apuntó que: "No debemos perder de vista un valor que no puede superar la virtualidad, que es la construcción social a partir del acercamiento de las personas. La construcción de una
Figura 1. Ciudad de México: índice de marginación, ubicación de universidades y residencia de estudiantes, basado en el mapa "Grado de marginación urbana a nivel áreas geoestadísticas básicas (AGEB) urbana" del Consejo Nacional de Población (CONAPO), 2010, y datos recolectados en el Tiud (dibujado por López y Ruiz, 2021).

Nota. Mapa que muestra la ubicación de las universidades participantes, así como los lugares de proveniencia de algunas estudiantes del TiUD 2020 y el grado de marginación de dichos lugares. 


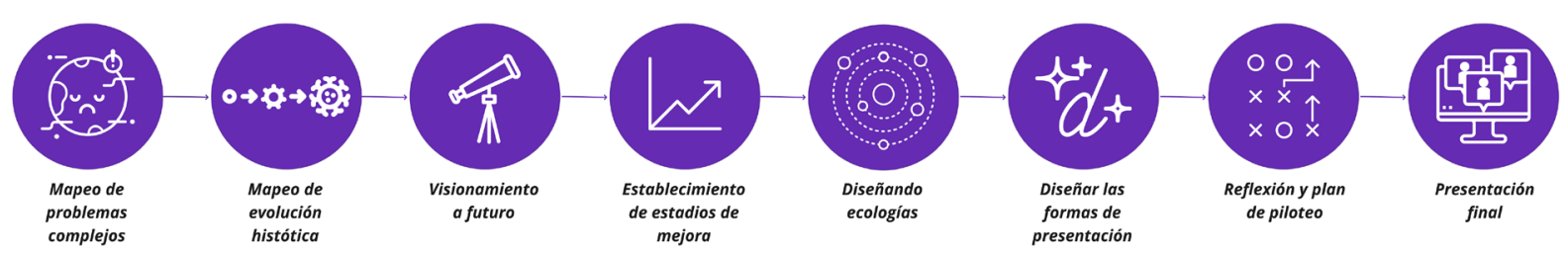

Figura 2. Programa general del TiUD 2020, con dos sesiones en promedio dedicadas a cada etapa, con base en el documento del TiUD 2020 (dibujado por Ruiz, 2021). misma y de su entorno y si eso funciona para todas igual, estamos haciendo una comunidad" (TiUD, 2020). No había que olvidar que la versión en línea es una modalidad durante la emergencia.

En concordancia con lo observado por Rossi et al. (2020), y a través del razonamiento abductivo que les caracteriza, durante la pandemia las diseñadoras lograron convertir imposibilidades en nuevas posibilidades. Las diseñadoras-docentes del TiUD fueron capaces de diseñar una versión del TiUD en modalidad de educación a distancia en línea, de manera sincrónica y con actividades asincrónicas (Bernardo \& Duarte, 2020), mediante las plataformas Zoom, Miro y Edmodo. El programa inició con un desayuno virtual el sábado 29 de agosto, al cual siguieron 16 sesiones de tres horas cada una, además de una sesión-fiesta organizada autogestivamente por las estudiantes y la presentación final (ver Figura 2). Cada sesión fue planeada y liderada por un equipo de tres diseñadoras-docentes (una titular y dos asistentes), quienes también eran apoyadas por la anfitriona de la sesión de Zoom y el resto de las docentes. Las estudiantes trabajaron predominantemente en equipos formados por áreas de interés, los cuales emergieron del análisis de diez temas originados por el contexto del confinamiento por Covid-19: educación, infraestructura, economía, medio ambiente, género, violencia, salud, cultura, alimentación y relaciones. Además, se diseñaron dinámicas de negociación entre equipos que les permitieron generar conjuntamente un manifiesto, una visión de futuro y una línea del tiempo. Las negociaciones interequipos también fueron un pretexto para permitir a las estudiantes relacionarse con más colegas.

Cada proyecto estudiantil consistía en una estrategia para lograr el futuro común para el 2100 desde una perspectiva particular. En conjunto, el TiUD 2020 generó un sistema de proyectos con el potencial de transformar la Ciudad de México. Como entregables finales, cada equipo generó los materiales solicitados por el Desafío Latinoamericano Index Award + UDD, en la convocatoria Diseño Responde (The Index Project, 2020), además de un llamado a la acción.

\section{Metodología}

El equipo de investigación fue integrado por cuatro alumnas del Posgrado en Diseño Industrial (PDI) de la Universidad Nacional Autónoma de México y una diseñadora-docente del TiUD ${ }^{13}$. Este equipo se conformó para dotar a 


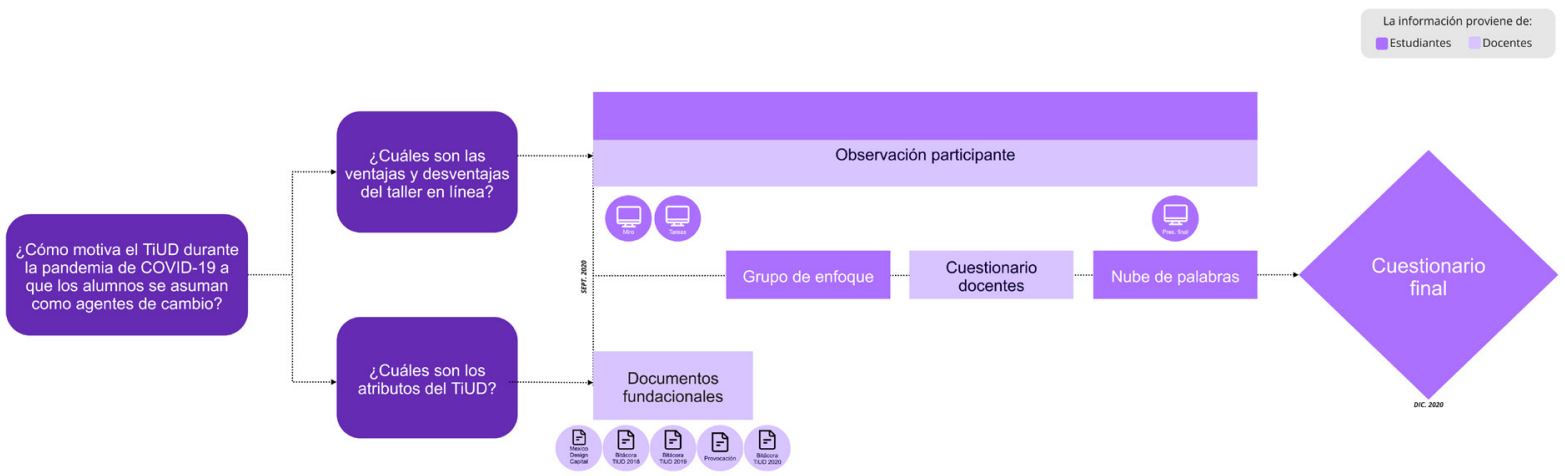

la investigación de mayor objetividad al obtener diferentes puntos de vista sobre el trabajo en las sesiones del TiUD.

Para el equipo de investigación, fue importante cotejar si los atributos plasmados en los materiales del TiuD coincidían con las percepciones de las docentes y especialmente, si éstos eran debidamente transmitidos hacia las estudiantes; de tal forma que algunas etapas de la investigación se desarrollaron de forma secuencial y estuvieron informadas por las etapas previas (ver Figura 3).

\section{Revisión Documental}

Se revisaron diversos documentos del TiUD, desde su fundación en el año 2018 hasta la última edición en 2020. Esto permitió la definición de los objetivos anteriormente mencionados y de los principales atributos como: colaboración, comunidad, lo común, experiencias alternativas de aprendizaje, una perspectiva profunda del Diseño, el activismo, la sostenibilidad y la transformación. Esta información se utilizó como base para el Diseño de los subsecuentes instrumentos de investigación.

\section{Observación Participante}

De manera sistemática, el equipo de investigación del PDI observó las dieciséis sesiones realizadas por medio de la plataforma Zoom. Esto permitió registrar las presentaciones e instrucciones de las diseñadoras-docentes, y el trabajo en equipo que las estudiantes realizaron por breakout rooms $s^{14}$ y el trabajo hecho en los tableros de Miro. La participación del equipo del PDI no fue neutral, sino que influyó en el desarrollo del curso al participar abiertamente en las discusiones de los equipos y en ocasiones suplir a alguna diseñadora-docente. Cabe resaltar que, también de las impresiones recolectadas del equipo PDI, se recolectaron aprendizajes y se extrajeron conclusiones.

\section{Percepciones de las estudiantes}

El equipo del PDI aplicó una entrevista grupal a una muestra conformada por cuatro equipos del TiUD, con el fin de abarcar un espectro amplio del desempeño generacional: a) un equipo con desempeño sobresaliente, b) dos equipos con desempeño regular y c) un equipo con desempeño deficiente. Dichos equipos fueron seleccionados con ayuda de las diseñadorasdocentes. La información resultante del análisis de esta herramienta se utilizó en el Diseño del cuestionario para las diseñadoras-docentes, una nube de palabras y el cuestionario final.
Figura 3. Metodología de investigación (dibujado por Ruiz, 2021)

Nota. Algunas herramientas se aplicaron de forma paralela y otras de manera secuencial. Algunas se diseñaron para conocer las percepciones de las docentes, otras de las estudiantes y otras las de ambos grupos.

13. A partir de aquí, diferenciamos equipo PDI, cuando únicamente fue un trabajo de las estudiantes del PDI, de equipo de investigación, cuando se incluye también la diseñadoradocente del TiUD.

14. Salas pequeñas por grupos de trabajo en la plataforma de Zoom. 


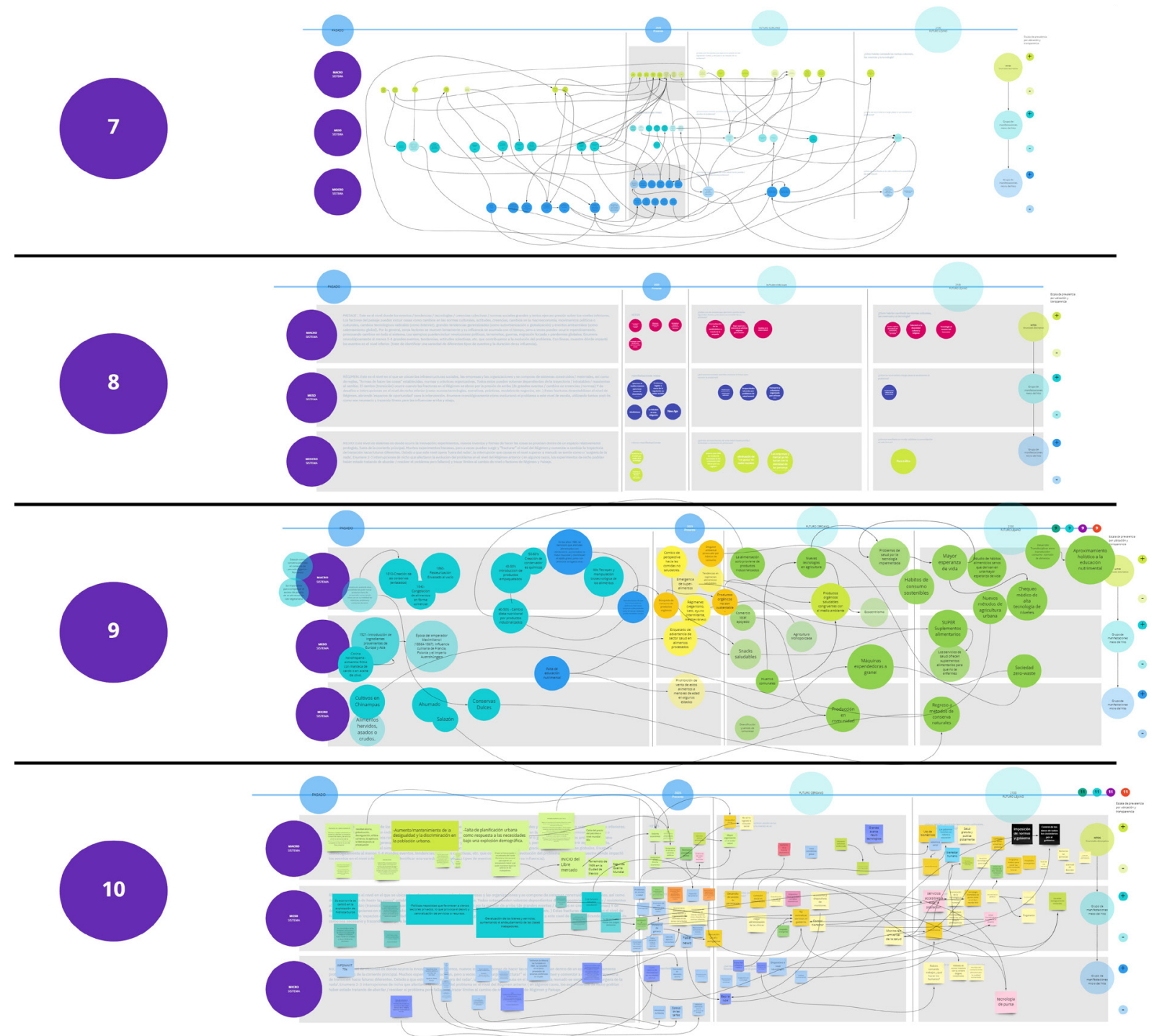

Figura 4. Ejemplo de interacción de los equipos en plataforma Miro (captura de pantalla, 30 de septiembre de 2020)

Nota. En la imagen se muestra un fragmento de una dinámica de identificación de hitos comunes y no comunes en las líneas del tiempo de los proyectos.

\section{Percepciones de las diseñadoras-docentes}

Con base en los resultados que arrojaron las dos herramientas anteriormente mencionadas, se diseñó un cuestionario que consistió mayoritariamente en preguntas abiertas, para recabar las percepciones de las diseñadoras-docentes. El cuestionario fue respondido por nueve de las doce diseñadoras-docentes provenientes de las cinco universidades participantes. Los resultados reflejaron una amplia concordancia con los documentos del TiUD, por lo cual esta información se utilizó en el Diseño del cuestionario aplicado a las alumnas en la fase final de esta investigación.

\section{Nube de palabras}

La nube de palabras fue una actividad desarrollada durante la presentación final transmitida de manera simultánea a través de Zoom, Youtube y Facebook Live del TiUD. Se formularon siete preguntas abiertas que debían contestarse con máximo dos palabras. Una vez terminada la encuesta, cada nube de respuesta fue proyectada a toda la audiencia. 


\begin{tabular}{|c|c|c|c|}
\hline Sección & Número de preguntas & Tipo de preguntas & Información obtenida \\
\hline \multirow{3}{*}{$\begin{array}{l}\text { 1. Introducción } \\
\text { "Hasta pronto } \\
\text { TiUD" }\end{array}$} & 2 & Opción múltiple & Datos demográficos \\
\hline & 3 & $\begin{array}{l}\text { Cerrada (opción } \\
\text { múltiple) }\end{array}$ & Motivaciones \\
\hline & 1 & Abierta & Definiciones de agente de cambio \\
\hline $\begin{array}{l}\text { 2. "Ventajas } \\
\text { y retos" }\end{array}$ & 18 & $\begin{array}{l}\text { Escalas de puntuación } \\
\text { por íntervalos }\end{array}$ & $\begin{array}{l}\text { Grado de dificultad de las tareas con } \\
\text { respecto a la virtualidad e interacción } \\
\text { con docentes y alumnos }\end{array}$ \\
\hline \multirow{2}{*}{$\begin{array}{l}\text { 3. "Reflexiones } \\
\text { finales" }\end{array}$} & 3 & Opción múltiple & $\begin{array}{c}\text { Opinión sobre el rol del docente y la } \\
\text { versión óptima del TiUD }\end{array}$ \\
\hline & 1 & Abierta & $\begin{array}{l}\text { Razones por las que se considera o no } \\
\text { el TiUD un hito en su vida }\end{array}$ \\
\hline
\end{tabular}

Figura 5. Síntesis del Diseño del cuestionario final a estudiantes (dibujado por Contreras y Ruiz, 2021).

\section{Cuestionario final a estudiantes}

Finalmente, con la información que arrojaron las diferentes herramientas, se formuló un cuestionario final que se aplicó a las alumnas el último día del semestre. Este consistió en 30 preguntas divididas en 3 secciones: a) Introducción; b) ventajas y desventajas; c) reflexiones finales (ver Figura 5). Este cuestionario incluyó preguntas que también formaron parte del cuestionario aplicado a las diseñadoras-docentes descritas anteriormente.

\section{Resultados y discusión}

En esta sección, los resultados de la observación participante se contrastaron con la información recabada mediante los instrumentos aplicados a los dos grupos de participantes: docentes y estudiantes. Posteriormente, se contrastaron las perspectivas de los dos grupos con el fin de:

1. Identificar si existe congruencia entre los atributos propuestos por las diseñadoras del TiUD (docentes) y las percepciones de las estudiantes de la versión en línea del taller.

2. Identificar las ventajas y desventajas que pudieron suscitarse en esta versión del TiUD, para generar recomendaciones en el futuro.

3. Identificar lecciones aprendidas que sean extensivas a otras experiencias educativas a distancia durante la emergencia por Covid-19.

\section{Diseñadoras-docentes}

Además de ser sujetos de la observación participante durante todo el semestre, en la mitad del curso se aplicó el cuestionario a las diseñadoras-docentes.

Todas las que habían estado presentes desde su fundación, respondieron que el Tiud es un hito en su vida, y dan motivos que pueden clasificarse dentro de las categorías de vocación, afiliación o ambos. La vocación se refiere a que, a nivel individual, las docentes identifican en el TiUD una oportunidad para llevar a cabo una acción transformadora. La categoría de afiliación se refiere a que encuentran en sus colegas tanto docentes como estudiantes, una comunidad o gremio, con la cual puedan ejercer dicha acción. Por su parte, las diseñadorasdocentes que después se involucraron al Tiud también encuentran estas oportunidades a nivel individual y colectivo. Solamente una persona piensa que es demasiado pronto como para saber si el Tiud es un hito en su vida. La aproximación al TiUD como generador de comunidad o gremio, es congruente con la forma en la cual las diseñadoras-docentes perciben su rol en 
15. Algunas universidades abrieron el curso a estudiantes de otras carreras, por lo cual se contó también con algunas estudiantes de ingeniería. el TiuD. Ninguna considera ejercer un rol tradicional, sino que se asumen como guías, tutoras o facilitadoras.

La información que se extrajo de las preguntas relacionadas con cómo experimentaban las diseñadoras-docentes la versión en línea del TiUD, fue clasificada bajo las siguientes categorías: a) la presencialidad y la virtualidad, b) fallas y mejoras en la comunicación, c) manejo del tiempo y d) documentación.

a. Presencialidad y virtualidad. Se extrañó especialmente la convivencia presencial y la posibilidad de realizar proyectos reales en y con comunidades de la CDMX; no obstante, la virtualidad permitió la participación de estudiantes y expertas que se encontraban en otros estados y países y a las estudiantes, que habitan por toda la ciudad, les permitió reunirse más veces fuera de los horarios del TiuD.

b. Fallas y mejoras en la comunicación. La comunicación docentes-estudiantes se dificulta en la modalidad en línea porque muchas veces, la docente no es capaz de leer la reacción de las estudiantes; entre docentes se dificulta principalmente por el número excesivo de canales de comunicación utilizados de manera simultánea. Paradójicamente, la virtualidad también ofreció ventajas en la comunicación, como la provisión de un espacio íntimo para los trabajos en grupo mediante Breakout Rooms.

c. Manejo del tiempo. La versión en línea requirió un tiempo excesivo de planificación, pero derivó en una programación mucho más cuidada que la de versiones anteriores.

d.Documentación. Además, se advierte que el uso de plataformas digitales permitió a las docentes identificar el progreso de las estudiantes en tiempo real, así como documentarlo de manera exhaustiva.

Finalmente, con pregunta directa, se corroboró que todas las diseñadorasdocentes perciben que una versión ideal del TiuD en el futuro integraría actividades presenciales y virtuales.

\section{Estudiantes ${ }^{15}$}

Toda la población estudiantil fue sujeta a la observación participante, en tanto una muestra (4/10 equipos) participó en los grupos focales que sirvieron como base para la nube de palabras, aplicada a 58 estudiantes. Todo lo anterior, más la información recabada del cuestionario aplicado a las diseñadoras-docentes, sirvió como base para la construcción del cuestionario a estudiantes; el cual fue respondido por 41 de los 58 participantes.

De la información recabada en este estudio a través de los grupos focales, se advierte que no se trabaja con una muestra aleatoria, sino con una muestra autoseleccionada, pues en sus procesos de selección, el TiUD convoca y atrae a un perfil muy particular de estudiantes de Diseño: aquel que en sus últimos semestres de la universidad se encuentra un tanto decepcionado con la práctica tradicional del Diseño y se encuentra abierto a la colaboración interuniversitaria, con la intención de contribuir activamente en el combate a las problemáticas más complejas de la actualidad. En el ejemplo a continuación, la entrevistadora pregunta a una alumna, ¿qué te motivó a participar en el TiUD? 
Yo tengo varias compañeras que participaron en la edición pasada, entonces después de su participación yo noté que estaban más entusiasmadas y tenían comentarios más positivos. Yo quise preguntarles, qué había pasado con ellas y pues me contaron del TiUD y por eso me animé a participar. (Participante 3 de la encuesta grupal bı)

Por otra parte, el cuestionario final logró constatar esta suposición, toda vez que las respuestas (de opción múltiple) más seleccionadas fueron: a) colaborar con otras escuelas (31/41); b) me pareció una propuesta interesante (22/41); c) explorar nuevas formas de hacer Diseño (20/41); d) aprender sobre otros enfoques de Diseño (20/41).

Adicionalmente, es importante señalar que si se presta atención a la participante que evaluó al TiUD de forma más negativa, en términos cuantitativos y cualitativos, fácilmente se advierte que es también la única que siempre tuvo carencias de infraestructura y conectividad, y señala que esto le impidió participar en muchas de las actividades del TiuD. Si esto se contrasta con los hallazgos de Adnan \& Anwar (2020), y con las proyecciones de deserción universitaria generadas por el Programa de Naciones Unidas para el Desarrollo (PNUD, 2020) y la Secretaría General de la UNAM (López \& Romero, 2020), debe reconocerse que la clase del TiUD 2020 estuvo preseleccionada también con base en su acceso a la infraestructura necesaria.

De esta discusión, se deriva la observación de que la autoselección de las participantes del TiUD puede influenciar directamente en el cumplimiento de los objetivos del curso. No es posible saber si un grupo aleatorio de estudiantes tendría una experiencia similar. En la mitad del semestre, las participantes de los grupos focales no reportaron una experiencia del todo gratificante y en el cuestionario final, el promedio general de las preguntas cerradas no es alto: 3.43 en una escala de 1-5, donde 5 fue el puntaje más alto. En concordancia con lo sugerido por la UNESCO (2019), se observa que el grupo de participantes que en el cuestionario final asignó las calificaciones más bajas también ofreció reflexiones profundas sobre su aprendizaje, por ejemplo:

En cuanto al Diseño, es un hito pues valoro mucho integrar a mis conocimientos el factor TIEMPO así como el Diseño de futuros o Diseño de transición. En cuanto a mi desarrollo personal también lo fue, pues mi experiencia dentro del equipo fue realmente difícil, lo que me lleva valorar más y trabajar desde el principio por una buena dinámica de equipo, impulsando desde el comienzo a que exista una buena convivencia y una buena participación de todas y todos los integrantes. (Participante A34)

Es decir, la participación transformadora no necesariamente corresponde con una experiencia completamente placentera. Aun cuando el promedio general del cuestionario fue ordinario, cerca del $95 \%$ consideró que el TiUD es un hito en su vida. Cuando se les preguntó por qué, la respuesta más frecuente fue que a partir del TiUD concibieron al Diseño de manera distinta (objetivo I del TiUD), seguida de respuestas relativas a las relaciones significativas construidas con sus compañeras y docentes (objetivo 3). Más del $90 \%$ de las encuestadas respondió que se considera así misma como agente de cambio, o cree que está en proceso de serlo (objetivo 2). 
Figura 6. Tabla que sintetiza los resultados de todas las preguntas cerradas del cuestionario, algunas de las abiertas y la evaluación que el equipo del PDI hizo de los proyectos finales (dibujado por Ruiz, 2021).

Nota. Las filas muestran las respuestas de cada participante, los participantes están organizados por equipo de trabajo y los equipos de trabajo, por problemática a tratar. Las columnas muestran las preguntas, organizadas por tema. La primera columna de izquierda a derecha sintetiza la cantidad de estudiantes que respondieron el cuestionario vs. la cantidad de integrantes en el equipo. Abajo, el promedio general de las preguntas abiertas. Las estrellas corresponden a la evaluación del equipo del PDI de cada uno de los cuatro rubros establecidos con base en las instrucciones de las docentes.

Las barras muestran los promedios por sección y el promedio general. La columna en el extremo derecho muestra las condiciones de infraestructura de cada estudiante (entre más larga sea la barra, mejores las condiciones de infraestructura).
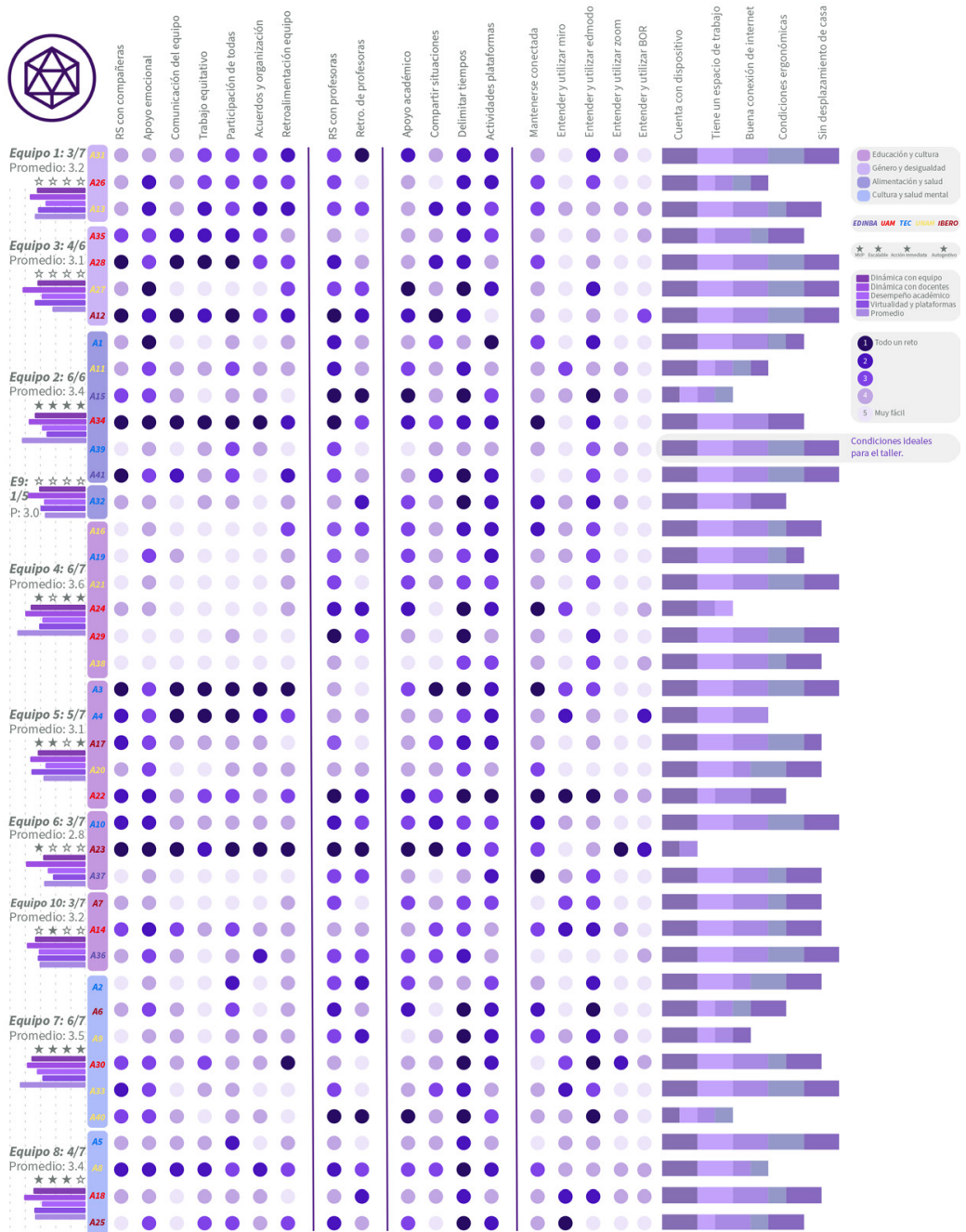

Desde la observación participante se advierte que, en general, cuando la dinámica del equipo es positiva (por ejemplo, la comunicación fluye, son capaces de dividirse las tareas de forma equitativa, etc.), los resultados del curso, parciales y finales, cumplen mejor con los requisitos planteados por las docentes. La comparación entre el cuestionario final para las estudiantes y un análisis de sus proyectos basado en las presentaciones finales arroja datos importantes. Los equipos 7,4 y 2 fueron los que mejor evaluaron su dinámica de equipo en el TiUD; junto con el equipo 8 , estos tres presentaron los proyectos mejor evaluados ${ }^{16}$ por el equipo del PDI (ver Figura 6). La mayor parte de las integrantes de estos equipos (6/7) respondieron el cuestionario, lo cual contrasta con el equipo 9 donde solamente una integrante de seis respondió. El desempeño de este equipo fue descrito como deficiente desde la mitad del curso, a raíz de un reporte directo en sus dinámicas de equipo; de ahí que fuera seleccionado para el grupo focal, al cual también faltaron participantes. Aunque los resultados del cuestionario de este equipo no son representativos, el ausentismo consistente refleja una dinámica complicada del equipo. 


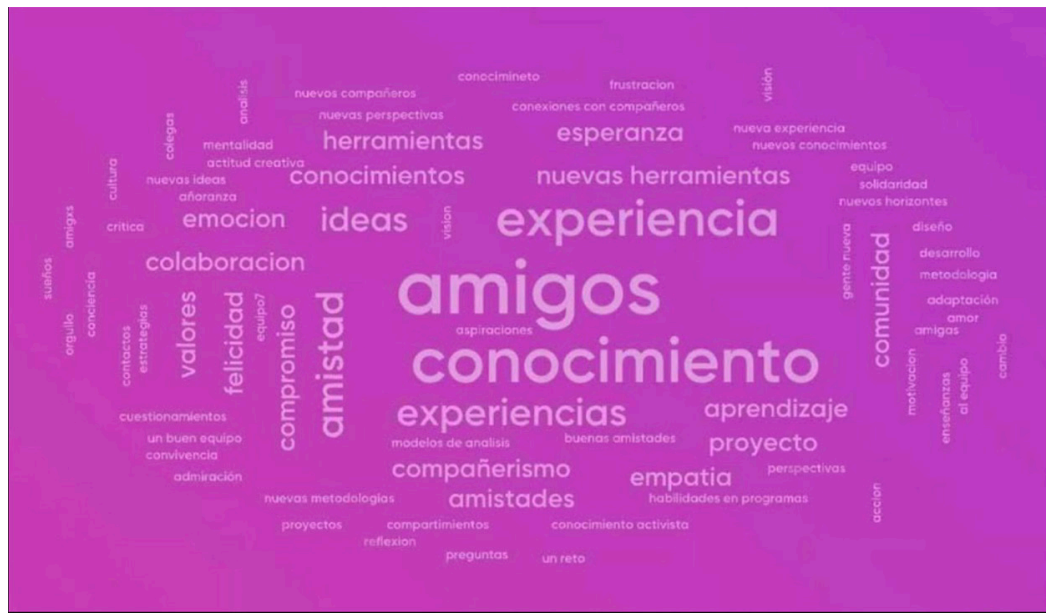

Independientemente del rol que las relaciones sociales significativas puedan jugar en el desarrollo de los proyectos, tanto los resultados de las nubes de palabras como las respuestas recabadas de forma abierta desde el cuestionario final muestran que las estudiantes, perciben a este tipo de relaciones como uno de los atributos más valiosos del TiUD. Cuando en la nube de palabras se les preguntó “Lo que me llevo del TiUD es...", las palabras más utilizadas fueron: amigos, amistad, comunicación y trabajo en equipo (ver Figura 7).

Además, en el cuestionario se obtuvieron respuestas como las siguientes:

- "Conocí a personas hermosas y logré entender el Diseño desde otra perspectiva" (Participante A9).

- "Marca un aprendizaje y un hecho en el que mi perspectiva y la manera en que puedo actuar es distinta. Me demuestra que puedo aportar mucho incluso con acciones pequeñas. Es un hito porque tomé una acción real y conocí colegas clave en mi vida" (Participante A24).

- "Aprendí a salir de mi zona de confort como persona introvertida para poder crear un proyecto increíble y que sé que cumple con mi propósito de ayudar a la sociedad. Además de aprender a trabajar con gente que no conozco de una forma nueva y en un momento histórico con el mismo fin" (Participante A28).

- "Porque me enseñó la importancia de la investigación y las narrativas para generar ideas de proyectos adecuados a mi realidad y pude conocer gente increíble entre profes y otras estudiantes y ya siento que las quiero mucho" (Participante A32).

\section{Contraste de perspectivas}

Con la finalidad de evaluar la congruencia entre lo que las diseñadorasdocentes quisieron transmitir y lo que las alumnas aprendieron, algunas preguntas fueron aplicadas a ambos grupos. Comprobamos que, tanto diseñadoras-docentes, como las alumnas del TiUD, interiorizaron los objetivos de aprendizaje planteados al principio del taller. Los motivos indicados para participar en el TiUD son muy parecidos entre ambas poblaciones tanto a nivel individual como colectivo: para todas es clara la estructura relativamente horizontal y la gran mayoría lo considera un hito en su vida. Cuando se les preguntó qué era o entendían por el concepto
Figura 7. Ejemplo de nube de palabras en aplicación Mentimeter (captura de pantalla, sábado 5 de diciembre de 2020).

16. Se advierte también que las instrucciones generadas por las diseñadoras-docentes fueron ambiguas, así que, al menos de forma comparativa y de acuerdo a los criterios definidos por el equipo del PDI (replicabilidad, escalabilidad, acción inmediata, autogestivo), algunos equipos que tuvieron un buen proceso, no llegaron al llamado a la acción. 
17. Por motivos como tener experiencia

profesional en la docencia, haber participado

en ediciones anteriores del TiUD, que la

investigación que llevan a cabo en el posgrado

está relacionada con los valores del TiUD. colaboración, ambos grupos, contestaron escuchar y compañerismo/ compañeros. En una especie de conversación, por medio de la nube de palabras, las alumnas escogieron la palabra compromiso, mientras que las docentes lo describieron en una pregunta abierta como una actividad en la que se participa equitativamente y se comparten experiencias. Además, para todas quedó claro que esta fue una versión del TiUD en emergencia, pero que la versión óptima integraría algunos aspectos presenciales y otros virtuales.

Adicionalmente, se encontró que la inserción de un grupo autoseleccionado ${ }^{17}$ de estudiantes de la Maestría en Diseño Industrial de la UNAM, fue y puede ser aún más benéfico para la dinámica de las próximas versiones del TiUD, independientemente de la modalidad. Las integrantes de este equipo reportan haber desarrollado el papel de intermediario, cercano a un consejero, cuando extendieron apoyo y orientación a las alumnas que lo solicitaron durante las observaciones participantes; pero también cumplieron funciones similares a las de una mediadora al recopilar información importante sobre la dinámica de los equipos y el desarrollo de la sesión. Es gracias a este nuevo rol, que las diseñadoras-docentes pudieron redirigir las sesiones y brindar atención especial a aquellos equipos, con dinámicas internas complicadas.

\section{Conclusiones}

El TiUD es una iniciativa educativa que busca que las estudiantes entiendan el Diseño como una poderosa herramienta para la transformación hacia futuros más justos y sostenibles y que, a través de experiencias profundas de aprendizaje logren asumirse como agentes de cambio tanto individual como colectivamente. Durante la emergencia común por Covid-19, posiblemente debido a la homogeneidad en actitudes y valores presente en la población que conforma su comunidad, el TiUD 2020, en una versión a distancia en línea logró cumplir con sus objetivos. No obstante, estudiantes y diseñadoras-docentes coinciden en que ésa no es la versión ideal, pues la virtualidad imposibilitó algunos aspectos invaluables de la socialización presencial. Por otra parte, la virtualidad acortó distancias, y permitió la interacción profunda y continua de quienes normalmente no coincidirían en la enorme ciudad que habitan. Adicionalmente, se hizo posible la inclusión de nuevos roles de participación y modalidades de trabajo.

Por lo tanto, una versión ideal postpandemia del TiUD combinaría sesiones presenciales y visitas a contextos reales con actividades virtuales, y aseguraría que todas las participantes tengan acceso a las plataformas digitales; de otra forma, el TiUD atentaría contra su propia concepción de lo común. Finalmente, podría contarse con estudiantes de maestría, en el rol de hermanas mayores, que actúen como un puente entre las docentes y las estudiantes del TiUD.

El potencial transformador del Diseño sólo puede detonarse desde la colectividad, porque "la acción individual no organizada, sin importar cuán bien sustentada esté, puede ser poco eficaz para lograr las metas de transformación" (UNESCO, 2020, p. 4). Las estudiantes refirieron que la mayor contribución del TiUD virtual es que, durante la emergencia común, les permitió compartir experiencias profundas que incentivaron el desarrollo de 
relaciones significativas, e incluso la emergencia de la amistad. Se concluye que, si la socialización se asume como un objetivo principal de un curso a distancia en línea, es posible fomentarla. Esta socialización no ocurrirá de forma óptima y no pretende reemplazar la interacción presencial. No obstante, en el momento histórico que vivimos, la principal contribución de este estudio es sugerir la integración de actividades que faciliten la socialización, durante y después de la emergencia, como aspecto prioritario de la educación transformadora en todas las disciplinas y en todos los niveles. 


\section{Referencias}

Adnan, M. \& Anwar, K. (2020). Online Learning amid the Covid-19 Pandemic: Students' Perspectives. Journal of Pedagogical Sociology and Psychology, 2(1), 45-51.

Bernardo, N. \& Duarte, E. (2020). Design, education, and the online tech-pandemic. Strategic Design Research Journal, 13(3), 577-585. https://doi.org/10.4013/sdrj.2020.133.22

Boni, A., MacDonald, P. \& Peris, J. (2012). Cultivating engineers' humanity: Fostering cosmopolitanism in a Technical University. International Journal of Educational Development, 32(1), 179-186. https://doi.org/10.1016/j. ijedudev.2011.07.001

Bravo, Ú. \& Bohemia, E. (2020). Editorial “Alfabetización en Diseño para todos." RChD: creación y pensamiento, 5(8), 1-10. https://doi.org/10.5354/0719-837X.2020.57649

Buchanan, R. (1992). Wicked Problems in Design Thinking. Design Issues, 8(2), 5-21. https://doi.org/10.2307/1511637

CONEVAL. (2020). Informe de pobreza y evaluación 2020 : Ciudad de México. CONEvAL. https://www.coneval.org.

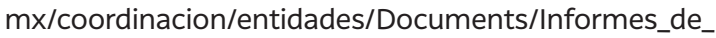
pobreza_y_evaluacion_2020_Documentos/Informe_ CDMX_2020.pdf

Consejo Nacional de Población. (2010). Índice de marginación urbana 2010. http://www.conapo.gob.mx/ es/CONAPo/Indice_de_marginacion_urbana_2010

Dussel, E. (2020, April 9). 2020: La Pandemia con Enrique Dussel. Ética y política [Aristegui Noticias]. https:// aristeguinoticias.com/0904/mexico/2020-lapandemia-con-enrique-dussel-etica-y-politica-en-vivo/

El Universal. (2019). Mejores Universidades [suplemento de periódico]. http://interactivo.eluniversal.com.mx/2019/ mejores-universidades/

Estalella, A., Rocha, J. \& Lafuente, A. (2013). Laboratorios de procomún: Experimentación, recursividad y activismo. Teknokultura, 10(1), 21-48.

Fernández, S. (2006). The Origins of Design Education in Latin America: From the hfg in Ulm to Globalization. Design Issues, 22(1), 3-19.

Freire, K. de M., Franzato, C. \& Remus, B. do N. (2020). Design amid Emergency. Strategic Design Research Journal, 13(3), 685-697. https://doi.org/10.4013/ sdrj.2020.133.30

Fuad-Luke, A. (2009). Design Activism: Beautiful Strangeness for a Sustainable World. Taylor and Francis.

Fuentes Nieva, R. \& Barrón Illescas, I. (2018, septiembre 25). Desigualdad en México. Seminario conEVAL. https://www.coneval.org.mx/Eventos/Documents/ Desigualdad-en-Mexico.pdf

Harari, Y. N. (2020, March 20). Yuval Noah Harari: The world after coronavirus. Financial Times. https://www.ft.com/ content/19d90308-6858-11ea-a3c9-1fe6fedcca75
Irwin, T. (2015). Transition Design: A Proposal for a New Area of Design Practice, Study, and Research. Design and Culture, $7(2)$, 229-246.

Irwin, T., Tonkinwise, C. \& Kossoff, G. (2020). Transition Design: An Educational Framework for Advancing the Study and Design of Sustainable Transitions. Cuadernos del Centro de Estudios en Diseño y Comunicación, 23(105), 31-65.

López, P. \& Romero, L. (2020, septiembre 28). Aumenta el riesgo de deserción en licenciatura y posgrado. Gaceta UNAM, 1372. https://www.gaceta.unam.mx/aumenta-elriesgo-de-desercion-en-licenciatura-y-posgrado/

Mextudia. (2020). Las Mejores Universidades en la Ciudad de México 2020. https://mextudia.com/rankings/ mejores-universidades-df-reforma/

Nussbaum, M. C. (2006). Education and Democratic Citizenship: Capabilities and Quality Education. Journal of Human Development, 7(3), 385-395. https://doi. org/10.1080/14649880600815974

Nussbaum, M. C. (2010). Not For Profit: Why Democracy Needs the Humanities. Princeton University Press.

Ostrom, E. (2002). Reformulating the Commons. Ambiente of Sociedade, Ano V(10), 1-22.

Programa de las Naciones Unidas para el Desarrollo. (2020). Desarrollo Humano y covid-19 en México: Desafíos para una recuperación sostenible. PNUD. https://www. mx.undp.org/content/mexico/es/home/library/poverty/ desarrollo-humano-y-covid-19-en-mexico-.html

Rezc,A.G.(2020, March 27). Las Mejores Universidades 2020. Reforma. Rossi, E., Nicolantonio, M. D., Ceschin, F., Mincolelli, G., Santos, A. dos, Kohtala, C., Jacques, E., Cipolla, C. \& Manzini, E. (2020). Design Contributions for the Covid-19 Global Emergency (Part 1): Empirical Approaches and First Solutions. Strategic Design Research Journal, 13(3), 294-311. https://doi.org/10.4013/sdrj.2020.133.01

Salgado, M. (s. f.). Diseño y diáspora. https://disenoydiaspora.org/ Secretaría de Desarrollo Económico. (2019). Guía para la Inversión 2019 CDMx. https://www.sedeco.cdmx.gob.mx/ storage/app/media/guia-para-la-inversion-2019-portal.pdf

Times Higher Education. (2020, junio 29). Latin America Rankings. Latin America University Rankings 2020. https:// www.timeshighereducation.com/world-universityrankings/2020/latin-america-university-rankings

The Index Project. (2020). Diseño Responde, Desafío Latinoamericano Index Award + UDD. https:// theindexproject.org/competitions/1/info

TiUD. (2018). Aprendizaje para el Diseño CDMX: Bitácora de Trabajo. TiUD. (2020). Taller Interuniversitario de Diseño: La emergencia de lo común.

TiUD. (2020a). Conversatorio interuniversitario: La emergencia (de lo) común. 
TiUD. (2020, ago 15). Conversatorio interuniversitario con Mariana Salgado y Ricardo Bello Bolio [Zoom].

UNESCO. (2019). Enseñando y aprendiendo para una participación transformadora. UNESCO. https:// unesdoc.unesco.org/ark:/48223/pfooo0368961_ spa?posinset $=90 \&$

Vázquez Pimentel, D. A., Dovalí Delgado, M. \& Jaramillo Molina, M. (s. f.). México Justo: Propuestas de políticas públicas para combatir la desigualdad. Oxfam México. https://www.oxfammexico.org/sites/default/files/ Informe\%20Me\%CC\%81xico-DAVOS-reducido.pdf Walker, M., McLean, M., Dison, A. \& Peppin-Vaughan, R. (2009). South African universities and human development: Towards a theorisation and operationalisation of professional capabilities for poverty reduction. International Journal of Educational Development, 29(6), 565-572. https://doi.org/10.1016/j. ijedudev.2009.03.002

Wilcox, P., Winn, S. \& Fyvie Gauld, M. (2005). 'It was nothing to do with the university, it was just the people': The role of social support in the first year experience of higher education. Studies in Higher Education, 30(6), 707722. https://doi.org/10.1080/03075070500340036 